\title{
Dispositivo alternativo para uso em controle de qualidade de monitores de imagem empregados no radiodiagnóstico médico
}

\author{
Alternative device for use in quality control of image monitors used in medical diagnostic \\ radiology \\ B. R. Mesquita ${ }^{1 *}$ L. A. P. Santos ${ }^{2}$; D. N. Souza ${ }^{1}$ \\ ${ }^{1}$ Departamento de Física,Universidade Federal de Sergipe, 49100-000, São Cristovão-Sergipe, Brasil \\ ${ }^{2}$ Centro Regional de Ciência Nuclear,Universidade Federal de Pernambuco, 50670-901, Recife-Pernambuco, Brasil
}

*bruno-delta10@hotmail.com

(Recebido em 02 de maio de 2018; aceito em 01 de outubro de 2018)

\begin{abstract}
Neste trabalho foram realizados testes de controle de qualidade em dispositivos de exibição de imagens (monitores) empregados em radiodiagnóstico médico e em monitores comuns. A avaliação foi realizada com base na curva característica da luminância em função da menor diferença detectável, curva DICOM GSDF. Nos testes foi empregado um fotodetector conectado a um eletrômetro como um método alternativo de medidor de luminância, a partir de grandezas elétricas. Com o auxílio de um LED no lugar do fotodetector, definindo-se o valor de ângulo sólido do fotodetector, foi possível a conversão de unidades de medida de iluminância em luminância, para a classificação dos monitores como primários ou secundários. Os seguintes testes foram realizados: reflexão difusa, reflexão especular, reflexão interna ou velamento, resolução, ruído, resposta de luminância e contraste e uniformidade de luminância. Para os testes, empregou-se imagens padrão teste denominadas de TG18 disponibilizadas pela Associação Americana dos Físicos em Medicina (AAPM). As recomendações de controle de qualidade para análise dos resultados foram baseadas no protocolo da própria AAPM e outros da Europa. Os softwares utilizados foram o Radiant DICOM e o Isyte Radiology da Phillips. O dispositivo alternativo possibilitou a classificação dos monitores, mostrando-se útil para a medição de luminância na ausência de fotômetro, que é o equipamento idealmente empregado para esse tipo de avaliação.
\end{abstract}

Palavras-chave: controle de qualidade, luminância, dispositivos de exibição, monitores de vídeo

In this work, quality control tests were performed on image display devices (monitors) used in medical radiodiagnosis and on common monitors. The evaluation was performed based on the luminance characteristic curve as a function of the smallest detectable difference, DICOM GSDF curve. A photodetector connected to an electrometer was used to the tests as an alternative method of luminance meter, with electrical quantities. With the aid of an LED in place of the photodetector and with the value of solid angle, the measurements were converted into photometric quantities to classify the monitors as primary or secondary. The following tests were performed: diffuse reflection, specular reflection, internal reflection or blurring, resolution, noise, luminance and contrast response and luminance uniformity. For the tests, standard test images of TG18 made available by the American Association of Physicists in Medicine (AAPM) were used. The quality control recommendations for the analysis of the results were based on the protocol of the AAPM itself and others from Europe. The software used was Radiant DICOM and Isyte Radiology of Phillips. The alternative device allowed the classification of the monitors, being useful for the measurement of luminance in the absence of photometer, which is the equipment ideally employed for this type of evaluation.

Keywords: quality control, luminance, display devices, video display

\section{INTRODUÇÃO}

O avanço da tecnologia tem aperfeiçoado os serviços de radiodiagnóstico médico, principalmente no que tange à radiologia digital. No que se refere ao controle de qualidade dos dispositivos de exibição de imagem utilizados em serviços de radiologia em geral, quando não se dispõe de equipamentos básicos para as avaliações requeridas, em algumas situações, podem ser empregadas maneiras alternativas para tais análises. Para isso, é necessário garantir previamente que os resultados obtidos por métodos alternativos sejam validados com o equipamento medidor 
da grandeza principal, que permite classificar o dispositivo de exibição como adequado ou não para o diagnóstico médico.

Em medicina, as imagens radiológicas resultam da interação de radiações ionizantes com o corpo humano. Essas imagens se apresentam com variações de tons de cinza. Para tons de cinza muito semelhantes, a visão humana apresenta dificuldade de percepção de diferenças entre esses tons. $\mathrm{O}$ padrão denominado DICOM GSDF, que pode ser definido como comunicação de imagem digital em medicina em função de exibição em tons de cinza, estabelece a quantidade de níveis de cinza com o número de bits de uma imagem digital e a menor diferença detectável (JND, do inglês, just noticiable difference) que garante a distinção entre os tons de cinza em uma imagem radiológica [1]. Os resultados de controle de qualidade em dispositivos de exibição são analisados com a finalidade de classificar esses dispositivos em primários e secundários, por meio da curva característica DICOM GSDF [1,2,3]. Os monitores primários são utilizados para prescrição de laudos médicos radiológicos, enquanto que os secundários são empregados para visualização de imagens radiológicas.

Os dados que possibilitam traçar as curvas características DICOM GSDF são obtidos por meio de análises de luminância dos monitores. Para tais análises, o instrumento tradicionalmente empregado é o fotômetro. Fotômetros para medições precisas de luminância costumam ser de elevado custo financeiro. Buscando uma alternativa que possa substituir o fotômetro, com uso de instrumentação de fácil execução, o objetivo deste trabalho foi analisar a qualidade de dispositivos de exibição de imagens (monitores) utilizados em radiodiagnóstico médico empregando um sistema composto por um LED (diodo emissor de luz, do inglês, light emitting diode) conectado a um eletrômetro. A título de comparação, também foram avaliados dois monitores de uso comum.

A luminância de um dispositivo de imagem representa a intensidade de luz refletida numa dada direção que o olho humano é capaz de visualizar, sendo definida como a intensidade luminosa por unidade de área de uma superfície em uma direção. A curva de luminância de um determinado monitor de diagnóstico deve estar de acordo com a curva padrão DICOM GSDF, apresentando valores de luminância em vários tons de cinza com dependência da iluminância do ambiente [4]. Tal padrão é a curva característica de luminância versus JND decorrente da medição de luminância, que está representada na figura 1, a partir de uma adaptação da Figura 45 do Report 03 [5].

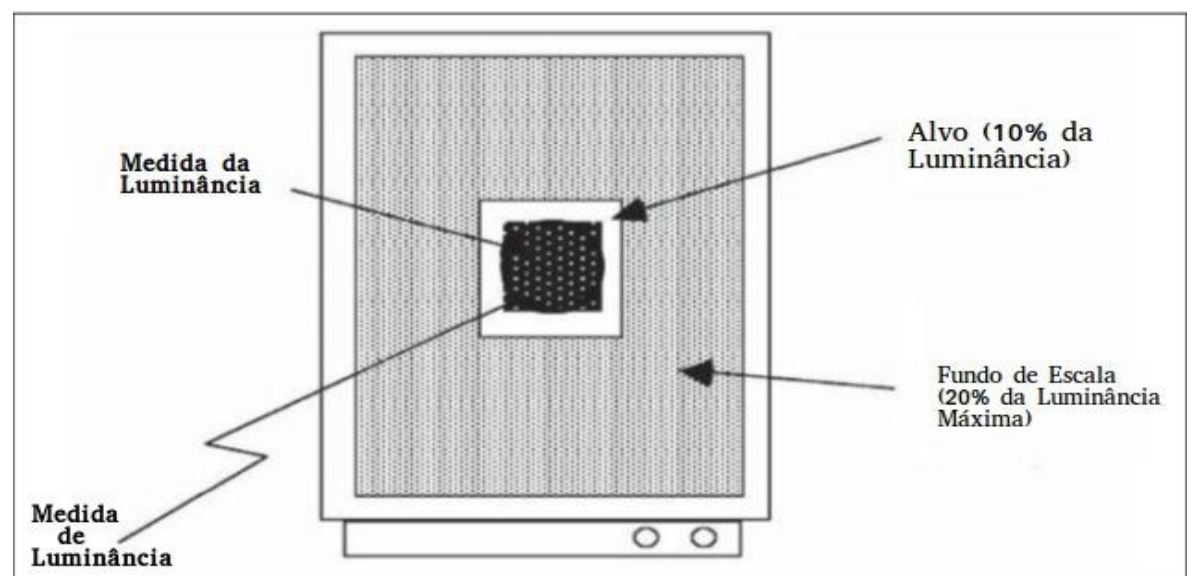

Figura 1: Representação esquemática da medida de luminância no TG18-LN, adaptada de [5].

O presente estudo teve como base protocolos publicados pela Associação Americana dos Físicos em Medicina (AAPM) [5], Protocolo Espanhol de Controle de Qualidade em Radiodiagnóstico (PECQR) [6] e Protocolo Europeu de Controle de Qualidade (PECQ) [7]. Para avaliação de monitores foram empregados processos qualitativos, com análises visuais considerando os padrões da imagem, e quantitativos, com a utilização de instrumentos de medição [8], considerando principalmente procedimentos do protocolo da AAPM.

Alguns estudos envolvendo controle de qualidade de dispositivos de exibição de imagens radiológicas, a partir de testes de verificação de desempenho, foram publicados anteriormente, a exemplo do realizado por Pinto et al. (2012) [9]. Porém, não são encontrados estudos relacionados 
com alternativas de medição de grandezas fotométricas que classificam os monitores aptos ou não para laudos médicos radiológicos.

Em relatos de literatura sobre avaliações de monitores das marcas Barco e Eizo, baseadas em testes de controle de qualidade, foi observado que monitores com resolução menor que 5 megapixels (5 MP) podem ser utilizados para o diagnóstico médico com base no teste de resposta de luminância e contraste [10,11].

No estudo realizado por Pinto et al. (2012) [9], a luminância de vinte e três monitores primários e vinte e dois monitores secundários, todos de cristal líquido (LCD), foi avaliada com um fotômetro, conforme protocolo da AAPM [5]. No estudo, os autores ressaltam que um plano de controle de qualidade adequado desses dispositivos de exibição tem impacto significativo no serviço prestado pelo profissional de saúde usuário de tais dispositivos. Os monitores devem fornecer imagens com densidade adequada de pixels para visualização em toda a área deles, com detalhamento espacial suficiente para distância de visualização normal de cerca de 30 a $60 \mathrm{~cm}$. Para o caso de monitor LCD, não é necessário avaliar a distorção geométrica por esse tipo de dispositivo possuir matriz fixa de pixels e não deformar a imagem [12].

\section{MATERIAL E MÉTODOS}

Foram avaliados quatro monitores, sendo dois da marca BARCO, empregados em radiodiagnóstico, e dois da marca SAMSUNG, de uso comum. Inicialmente, foram instalados nos computadores aos quais esses monitores estavam dedicados os softwares para a exibição e análise das imagens fornecidas pelo TG18 [5], no formato DICOM, para quantificação da qualidade dos monitores. Essas imagens padrões correspondem a oito bits (256 tons de cinza) com formato de 1.000 pixels, e a doze bits (4096 tons de cinza), com formato de 2.000 pixels.

Os protocolos de controle de qualidade exigem que não haja contribuição de fontes de luz externas nas imagens exibidas nos monitores do tipo primário. Isso, idealmente, significa ausência de luminosidade proveniente de fontes externas aos monitores, o que representa iluminância igual a zero lux. Nas salas em que foram avaliados os dispositivos de exibição não foi observado luminosidade externa.

Os softwares instalados para visualização das imagens teste foram o Radiant Dicom e o Isyte Radiology [1]. A luminância foi obtida por um método alternativo, para o qual é necessário definir o ângulo sólido do fotodetector para a conversão de unidades de medida de iluminância (lux) em luminância $\left(\mathrm{cd} / \mathrm{m}^{2}\right)$. Para a definição do ângulo foi utilizado um LED no lugar do fotodetector (figura 2). O ângulo sólido do LED de espectro amarelo, com uma estrutura circular, apresentou um valor de $0,1538 \mathrm{sr}$. A conversão de unidades no SI, de lux para $\mathrm{cd} / \mathrm{m}^{2}$, resultou em 1 lux $=$ $1 \mathrm{~cd} . \mathrm{sr} / \mathrm{m}^{2}$.

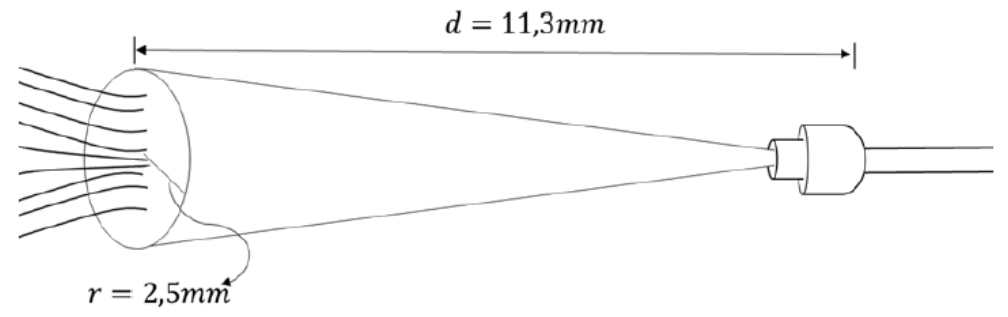

Figura 2: Representação esquemática do ângulo sólido do fotodetector, de autoria própria.

Os equipamentos utilizados nas medições foram: um luxímetro MLM-1020, um fotodetector de marca Infineon SFH 2505, um eletrômetro (medidor de corrente elétrica) e um luxímetro Minolta digital - fotômetro MT791 com um fator de conversão de 18,51.

No método alternativo com uso de eletrômetro foram realizadas medições de corrente elétrica em unidades de nano e microampère em 18 níveis de cinza das imagens padrão TG18-LN12, TG18UNL10 e TG18-UNL80. A luminância, o contraste e a uniformidade de luminância foram avaliados com o fotodetector posicionado no centro das imagens exibidas nos monitores.

Os monitores analisados foram da marca SAMSUNG (S20B300 e B2230) e BARCO (MDCC 4130 e MDRC 2124). Os das marcas BARCO apresentavam um tempo de uso de 292 e 878 horas, 
respectivamente, enquanto que os da marca SAMSUNG apresentaram um tempo de 180 horas. Para todos os monitores foi estabelecido um padrão único de $80 \%$ de brilho.

Os testes realizados para o controle de qualidade dos dispositivos de exibição foram: reflexão difusa, reflexão especular, reflexão interna ou de velamento, resolução, ruído, resposta de luminância e contraste e uniformidade de luminância. Esses testes estão descritos a seguir.

\subsection{Reflexão difusa}

No teste de reflexão difusa, os resultados obtidos são quantitativos, resultando na obtenção do coeficiente de reflexão difusa $\left(\mathrm{R}_{\mathrm{d}}\right)$. Esse coeficiente é definido como sendo a razão da luminância ambiente, que é a razão definida entre a luminância obtida com o fotodetector ou fotômetro encostado à tela do monitor desligado, ou em modo de economia de energia, e a iluminância ambiente obtida com o luxímetro a uma distância de $30 \mathrm{~cm}$ do monitor.

\subsection{Reflexão especular}

O teste de reflexão especular tem por finalidade aferir se há formação de imagens espelhadas sob a tela do monitor, que são resultados de reflexos de fontes externas, e se essas reflexões influenciam na imagem padrão TG18-AD exibida no monitor avaliado. Para a avaliação quantitativa desse teste são medidos os valores de luminância e do limiar de contraste, considerando-se o coeficiente de reflexão difusa e a iluminância ambiente.

\subsection{Reflexão interna ou de velamento}

O teste de reflexão interna ou de velamento determina a perda de informação devido à dispersão da luz da tela do monitor emitida a partir do interior do dispositivo. Para isso, examina-se se as estruturas que são visíveis no padrão TG18-GV utilizando-se um papel escuro com um orifício centrado e, em seguida, quantificam-se as estruturas visíveis no padrão TG18-GVN para que se possa classificar os monitores em primários ou secundários.

\subsection{Resolução}

Com o teste de resolução se verifica a capacidade do monitor em apresentar a menor estrutura possível de uma imagem radiológica. A imagem padrão empregada é a TG18-CX, que apresenta elementos do tamanho do pixel do monitor. Os padrões CX são a quantificação das estruturas, sendo essa uma nomenclatura especifica do teste. Para que os monitores sejam classificados como primários, a pontuação dos objetos CX deve estar entre 0 e 4 . Para os secundários, na visualização de imagens médicas, as pontuações dos objetos podem estar compreendidas entre 0 e 6.

\subsection{Ruído}

O teste de ruído é um informativo do comportamento da resposta de luminância do monitor, e em casos em que esse teste apresenta falhas, as respostas de luminância e de contraste devem ser imediatamente verificadas. Esse teste tem como finalidade avaliar a existência de artefatos ou pixels defeituosos que possam comprometer o diagnóstico de estruturas com um contraste de baixo nível. A imagem padrão empregada é a TG18-AFC, que é dividida em quatro quadrantes. Nos monitores primários serão visualizados pelo menos três dos quatro quadrantes em todos os pontos visíveis. Nos monitores de visualização, pelo menos dois dos quatro quadrantes precisam apresentar todos os pontos visíveis.

\subsection{Resposta de luminância e contraste}

No teste de resposta de luminância e contraste é estabelecida uma relação entre a luminância medida e os valores de entrada de um sistema padronizado, que consiste na luz produzida por um dispositivo de exibição no intervalo da luminância máxima e luminância mínima, juntamente com a contribuição fixa da luz ambiente refletida difusamente. $O$ valor da luminância máxima deve ser maior que $170 \mathrm{~cd} / \mathrm{m}^{2}$ para monitores primários e maior que $100 \mathrm{~cd} / \mathrm{m}^{2}$ para monitores secundários. 
A imagem padrão empregada no teste é a TG18-LN12, que corresponde a 18 imagens no padrão DICOM GSDF, sendo que a quantidade de pixels que compõem as imagens teste varia entre $0 \mathrm{e}$ 4080. As luminâncias obtidas nas imagens TG18-LN12-01 e TG18-LN12-18 correspondem à luminância mínima e à luminância máxima dos dispositivos, respectivamente. A luminância é obtida com o equipamento (fotômetro ou eletrômetro) encostado na tela do monitor no centro da imagem TG18-LN12 selecionada.

Para esse teste, ao final das medições, com os dados, foi definida uma curva teórica com todos os valores de luminância em vários tons de cinza nas dezoito imagens padrão com dependência da iluminância ambiente, sendo então a curva DICOM GSDF similar à figura 45 do Report 03 [5].

Na avaliação de controle de qualidade por meio do teste de uniformidade de luminância são utilizadas imagens padrões, especificadas com $10 \%$ e $80 \%$ de luminância máxima, que possibilitam verificar se há regiões na tela do monitor onde para um mesmo tom de cinza ocorre variação de intensidade luminosa. As imagens padrão para esse teste são TG18-UNL80 e TG18-UNL10. O GSDF foi obtido em vários locais das telas dos monitores com a finalidade de observar a uniformidade de luminância. As luminâncias nas quatro bordas e do centro não podem variar em mais de $30 \%$ para os monitores primários e $15 \%$ para os monitores secundários, e caso os valores obtidos estejam fora do GSDF aceitável, devem ser realizadas ações corretivas, com reparação ou substituição do dispositivo de exibição.

\section{RESULTADOS E DISCUSSÃO}

Para as avaliações dos monitores com grandezas elétricas e fotométricas, levou-se em consideração o tempo de uso de cada monitor. A seguir, estão discutidos os resultados para cada teste de controle de qualidade.

\subsection{Reflexão difusa}

A tabela 1 apresenta os resultados do teste de reflexão difusa obtidos com o eletrômetro e medidor de luminância (fotômetro).

Tabela 1: Valores de luminância e coeficiente de reflexão difusa (Rd) medidos nos equipamentos investigados.

\begin{tabular}{cccc}
\hline & \multicolumn{3}{c}{ Teste de reflexão difusa } \\
\cline { 2 - 4 } Marca/Modelo & Iluminância (lux) & $\begin{array}{c}\text { Luminância } \\
\text { ambiente }\left(\mathbf{c d} / \mathbf{m}^{2}\right)\end{array}$ & Rd (cd/m².lux) \\
\hline Samsung S20B300 & 9,25 & 0,176 & 0,019 \\
Samsung B2230 & 9,25 & 0,178 & 0,019 \\
Barco MDCC 4130 & 9,76 & 0,185 & 0,018 \\
Barco MDRC 2124 & 7,23 & 0,185 & 0,025 \\
\hline
\end{tabular}

De acordo com a tabela 1 , a luminância ambiente não ultrapassou o limite estabelecido de $2 / 3$ da luminância mínima $\left(1,30 \mathrm{~cd} / \mathrm{m}^{2}\right)$ dos monitores SAMSUNG. O coeficiente de reflexão difusa apresentou valores entre 0 e 1, o que significa que a iluminância ambiente está de acordo com os limites estabelecidos pelos protocolos de controle de qualidade e que não ocorreu perda de informação no teste de reflexão difusa, sendo possível visualizar as estruturas presentes na imagem digital.

Para os monitores da marca BARCO, a luminância ambiente apresentou valor de $0,185 \mathrm{~cd} / \mathrm{m}^{2}$. A diferença entre os valores do coeficiente de reflexão difusa está diretamente relacionada com o tempo de uso do monitor, bem como com a posição em que o monitor se encontrava na sala de laudo. Nesse caso, observa-se que o monitor Barco MDRC 2124 que possuía o maior tempo de uso (878 horas) apresentou o maior índice de coeficiente de reflexão difusa. No entanto, não houve interferência no contraste da imagem ou visualização de estruturas externas nas imagens médicas, o que significa que todos apresentaram um percentual de 66,6 \% da luminância com relação ao 
valor da luminância mínima do monitor. Esse percentual estimado é equivalente a $2 / 3$ da luminância mínima do monitor em relação à luminância ambiente.

\subsection{Reflexão especular}

No teste de reflexão especular, o coeficiente de reflexão é definido considerando-se a influência das grandezas medidas no monitor avaliado. O coeficiente de reflexão difusa ( $\mathrm{Rd}$ ) possui uma relação direta com o coeficiente de reflexão especular (Rs), levando em conta a iluminância ambiente e as estruturas espelhadas que podem ser visualizadas no monitor, que são resultantes de fontes de luz externas que incidem diretamente sobre o monitor. A tabela 2 apresenta os coeficientes obtidos considerando os valores de iluminância, luminância máxima e o limiar de contraste $(\mathrm{Ct})$.

Tabela 2: Características dos dipositivos de exibição de imagem no teste de reflexão especular (Rs).

\begin{tabular}{ccccc}
\hline & \multicolumn{3}{c}{ Teste de reflexão difusa } \\
\cline { 2 - 5 } Marca/Modelo & $\begin{array}{c}\text { Iluminância } \\
(\mathbf{l u x})\end{array}$ & $\begin{array}{c}\text { Luminância } \\
\text { mínima }\left(\mathbf{c d} / \mathbf{m}^{2}\right)\end{array}$ & $\mathbf{C}_{\mathbf{t}}$ & $\begin{array}{c}\mathbf{R s} \\
\left(\mathbf{c d} / \mathbf{m}^{2} \mathbf{l} \mathbf{l u x}\right)\end{array}$ \\
\hline Samsung S20B300 & 9,25 & 1,30 & 0,024 & 0,0112 \\
Samsung B2230 & 9,25 & 1,30 & 0,024 & 0,0112 \\
Barco MDCC 4130 & 5,52 & 0,74 & 0,024 & 0,011 \\
Barco MDRC 2124 & 7,23 & 0,55 & 0,024 & 0,006 \\
\hline
\end{tabular}

A luminância dos dipositivos da marca SAMSUNG foi obtida pelo com o eletrômetro (método alternativo), enquanto que os da marca BARCO foram avaliados com o luxímetro calibrado. Os resultados de luminância mínima e de limiar de contraste obedeceram ao que determina a AAPM, constatando-se que para monitores com uma maior resolução, mesmo limiar de contraste e com moderada variação de iluminância, os resultados de coeficiente de reflexão especular foram semelhantes entre si. Isso comprova que não houve interferência de fontes externas que dificultassem a visualização das informações contidas na imagem radiológica digital, pelo fato da iluminância ambiente medir zero lux. É necessário atenção com a posição em que se encontra o monitor em relação às fontes de luz, para que não haja interferência de luz ambiente no coeficiente de reflexão especular, o que causa formação de imagens espúrias que influenciam no contraste dos dispositivos. Essa interferência também causa uma iluminância inadequada, considerando-se os requisitos de controle de qualidade.

\subsection{Teste de reflexão interna ou de velamento}

A avaliação da qualidade dos dispositivos de exibição com base no teste de reflexão interna ou de velamento foi de fundamental importância para atestar a qualidade deles. Os resultados descritos nas tabelas 1 e 2 demonstram que em todos os dispositivos é possível observar a imagem padrão teste TG18-GV com cinco estruturas completamente visíveis, significando a ausência ou pouca quantidade de reflexão difusa e especular. Esse resultado assinala que o tempo de uso dos dispositivos não interferiu na qualidade da visualização das imagens e na quantificação das estruturas.

Em relação à imagem TG18-GVN, nos monitores da marca BARCO foram visualizadas cinco estruturas, o que os caracteriza como monitores primários. Nos monitores da marca SAMSUNG apenas três estruturas foram visualizadas, sendo esses classificados como monitores secundários. Em comparação com resultados apresentados por [11], os monitores aqui classificados como secundários apresentaram resultados comparáveis aos primários na questão da reflexão difusa, tendo todos atingido os limites estabelecidos.

\subsection{Resolução}

O teste de resolução permite verificar em uma imagem radiológica digital a menor quantidade de estruturas visíveis com ausência de iluminância ambiente, baixa presença de ruído ou iluminância reduzida. Esse teste tem a função de quantificar os padrões $C X$ em uma imagem 
exibida nos dispositivos de exibição. Os padrões CX são quantificações exclusivas do teste de resolução. Caso seja verificada reflexão difusa e especular, deve-se observar se essa prejudica a visualização dos padrões na imagem. Nos monitores da marca BARCO foi percebida a mesma quantidade de estruturas no centro da imagem, nas partes superior direita e esquerda, inferior esquerda e direita, confirmando o padrão $\mathrm{CX}=1$ visualizado no zoom de $100 \%$ nos monitores. Isso foi suficiente para classificar qualitativamente esses monitores como primários. Nos monitores da marca Samsung, visualizou-se as estruturas em todas as regiões com $C X=0$, o que os certifica como úteis para visualização de imagens médicas (monitores secundários).

\subsection{Ruído}

O teste de resposta de luminância e contraste está diretamente relacionado com o teste de ruído. Esse teste quantifica as estruturas de baixo contraste visíveis na imagem padrão teste TG18-AFC a partir dos limiares estabelecidos, para que os monitores sejam classificados como primários ou secundários, considerando um sistema visual humano com boa acuidade visual. A imagem padrão teste foi subdividida em quatro quadrantes $\left(1^{\circ}, 2^{\circ}, 3^{\circ}\right.$ e $\left.4^{\circ}\right)$. Nos monitores da marca BARCO foram observadas cinquenta e nove estruturas tanto no primeiro quanto no segundo quadrante e cinquenta e oito estruturas no terceiro quadrante; no quarto quadrante não se observou nenhuma estrutura, como esperado. Nesse teste se levou em consideração a presença de pixels defeituosos, o que faz com que o sistema visual humano não identifique todas estruturas. Nos monitores da marca SAMSUNG só foi possível verificar cinquenta e nove estruturas em dois dos quatro quadrantes. Esse resultado concorda com outros testes, classificando esses monitores como secundários.

\subsection{Resposta de luminância e contraste}

O teste de resposta de luminância e contraste tem o objetivo de averiguar os resultados de acordo com o GSDF e em mais ou menos dez por cento do JND, levando em conta os níveis de luminância nas dezoito imagens padrão TG18-LN12. Tal teste possibilitou obter a resposta de luminância, a curva característica de acordo com o JND e os valores de iluminância, considerando o fator de calibração obtido com o valor do ângulo sólido calculado anteriormente. A tabela 3 apresenta esses valores, os pixels para cada imagem padrão e os respectivos limiares de contraste (dL/L). Os resultados apresentados na tabela 3 para o monitor Barco MDCC 4130 são análogos aos obtidos no monitor Barco MDRC 2124.

No monitor Barco 4130 foi possível visualizar a imagem teste com qualidade no lado direito e esquerdo, não sendo observados pixels defeituosos nem reflexão especular que pudessem comprometer o diagnóstico médico. De acordo com os protocolos de controle de qualidade os valores de luminância máxima estão dentro dos limites estabelecidos para que o monitor seja classificado como primário. Esse monitor possuía um tempo de uso de 292 horas. Entretanto, a curva característica da figura 3 apresenta uma superposição da curva DICOM com mais ou menos dez por cento, o que comprova uma não conformidade com o padrão GSDF e os limites percentuais do JND. Não foram observados detalhes durante a avaliação que possam prejudicar o diagnóstico médico, mesmo com baixo coeficiente de reflexão especular e iluminância adequada no ambiente em que o dispositivo se encontrava. O monitor possuía uma resolução de 2 MP. A razão de luminância apresentou um valor equivalente a 813 e 643 para a razão de contraste. As diferenças em termos de reflexão especular relacionam o grau de comprometimento da imagem. Considerando-se a razão de contraste, esse dispositivo foi classificado como monitor secundário.

A curva característica da figura 4 se refere ao teste do monitor Barco MDRC 2124. Esse dispositivo de exibição é utilizado para exames de raios X, tomografia e hemodinâmica, possuindo resolução de 2,3 MP. A curva DICOM GSDF apresentou conformidade com os limiares do JND em mais ou menos dez por cento, evidenciado pelo crescimento dos valores de luminância nas medições na imagem padrão teste. A razão de luminância apresentou valor equivalente a 602 e a razão de contraste valor de 448 . O teste foi realizado em ambiente de sala de laudo com iluminância adequada. $\mathrm{O}$ baixo valor de coeficiente de reflexão especular também contribuiu para que a curva apresentasse um comportamento adequado. Considerando-se os valores de luminância máxima, razão de luminância e razão de contraste, esse dispositivo foi classificado como monitor primário. 
Tabela 3: Grandezas fotométricas para o monitor Barco MDCC 4130

\begin{tabular}{cccccc}
\hline & \multicolumn{3}{c}{ Teste de resposta de luminância e contraste } & & \\
\cline { 2 - 6 } Imagem Teste & $\begin{array}{c}\text { Valor de } \\
\text { pixels }\end{array}$ & Iluminância (lux) & Luminância (cd/m $\left.\mathbf{m}^{2}\right)$ & JND & dL/L \\
\hline TG18-LN12-01 & 0 & 0,05 & 0,74 & 59,60 & - \\
TG18-LN12-02 & 240 & 0,14 & 2,40 & 114,09 & 1,06 \\
TG18-LN12-03 & 480 & 0,28 & 4,99 & 161,21 & 0,70 \\
TG18-LN12-04 & 720 & 0,48 & 8,70 & 204,78 & 0,54 \\
TG18-LN12-05 & 960 & 0,75 & 13,69 & 245,70 & 0,45 \\
TG18-LN12-06 & 1200 & 1,12 & 20,54 & 286,22 & 0,40 \\
TG18-LN12-07 & 1440 & 1,6 & 29,43 & 325,16 & 0,36 \\
TG18-LN12-08 & 1680 & 2,23 & 41,09 & 363,71 & 0,33 \\
TG18-LN12-09 & 1920 & 3,06 & 56,45 & 402,40 & 0,32 \\
TG18-LN12-10 & 2160 & 4,19 & 77,37 & 442,53 & 0,31 \\
TG18-LN12-11 & 2400 & 5,55 & 102,54 & 479,73 & 0,28 \\
TG18-LN12-12 & 2640 & 7,33 & 135,49 & 517,62 & 0,28 \\
TG18-LN12-13 & 2880 & 9,63 & 178,07 & 555,71 & 0,27 \\
TG18-LN12-14 & 3120 & 12,4 & 229,34 & 591,70 & 0,25 \\
TG18-LN12-15 & 3360 & 15,9 & 294,13 & 627,67 & 0,25 \\
TG18-LN12-16 & 3600 & 20,3 & 375,58 & 663,50 & 0,24 \\
TG18-LN12-17 & 3840 & 25,6 & 473,68 & 697,88 & 0,23 \\
TG18-LN12-18 & 4080 & 32,3 & 597,71 & 732,65 & 0,23 \\
\hline
\end{tabular}

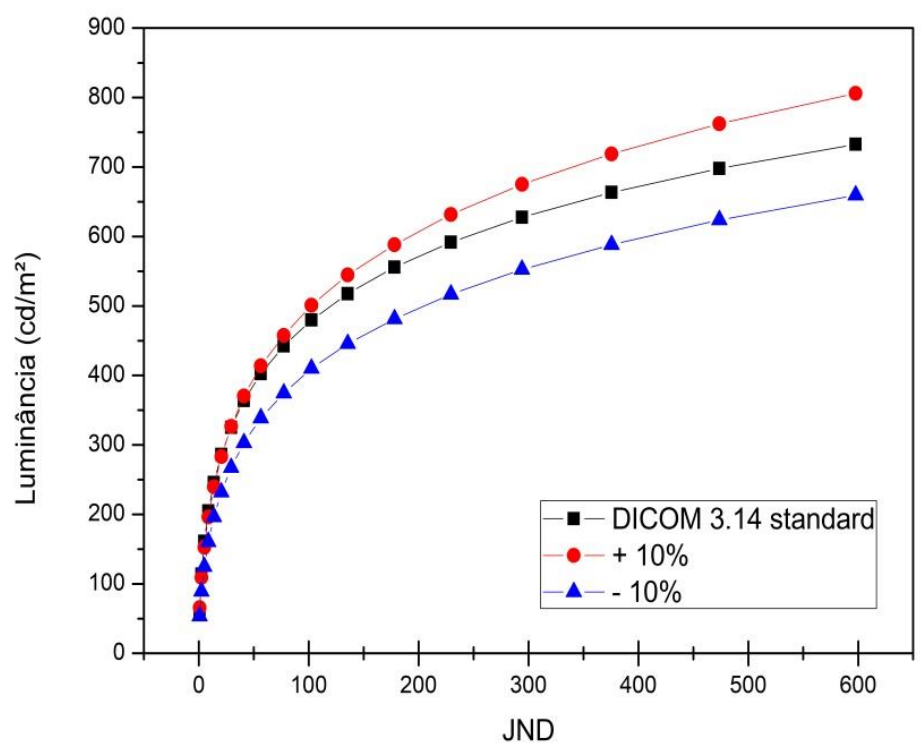

Figura 3: Curva característica DICOM GSDF da luminância versus JND com brilho de $80 \%$ para o monitor BARCO MDCC 4130. 


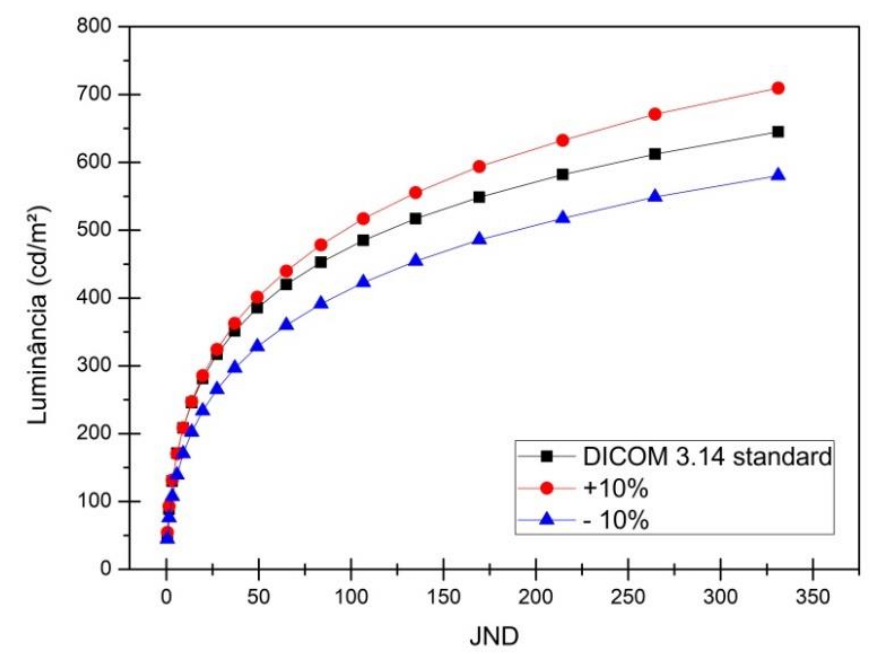

Figura 4: Curva característica DICOM GSDF da luminância versus JND com brilho de $80 \%$ para o monitor BARCO MDRC 2124.

O gráfico da figura 5 apresenta a resposta fotônica, que representa o comportamento da corrente elétrica versus potência elétrica aplicada ao dispositivo alternativo. A corrente elétrica não variou linearmente com a potência. O LED empregado emite luz alaranjada, com comprimento de onda constante, da ordem de $590 \mathrm{~nm}$. A temperatura ambiente manteve-se constante durante a realização das medições e com iluminância nula. A corrente elétrica inicial do componente utilizado apresentou valor de 1,0 nA. Ao aumentar a potência elétrica (Pe), observou-se que em comparação com a luminância, a corrente cresceu linearmente. Na análise dos monitores, o valor da corrente elétrica depende da quantidade de luz absorvida pelo fotodetector através das imagens padrão teste TG18-LN12 que foram utilizadas para obtenção dos valores de luminância.

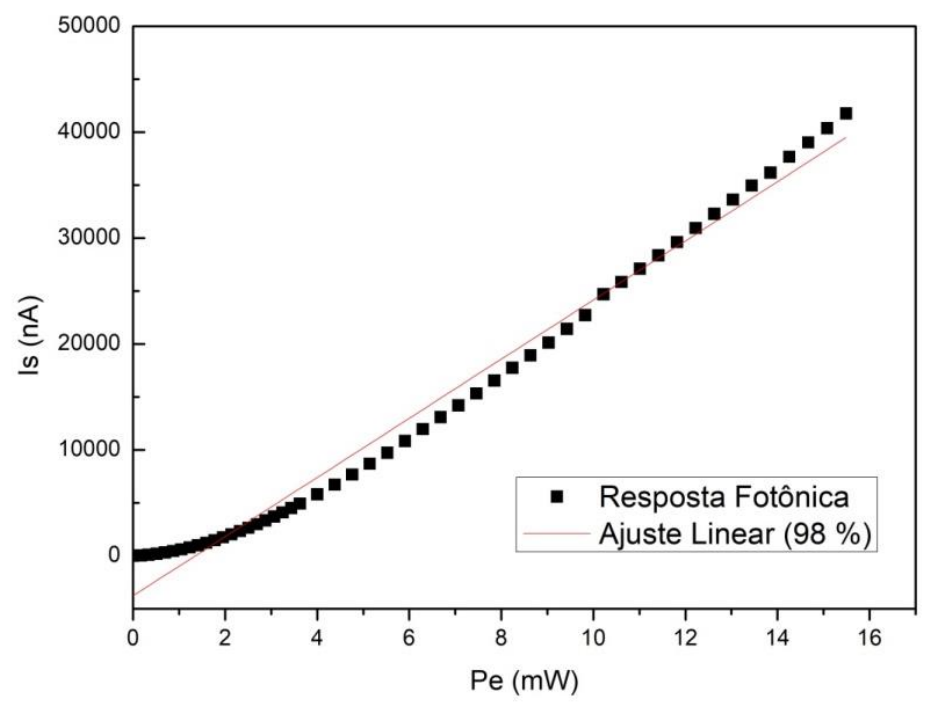

Figura 5: Resposta fotônica com brilho de $100 \%$ para o monitor SAMSUNG S20B300. 
Para o teste de resposta de luminância e contraste foi empregado o dispositivo alternativo para a obtenção de valores de luminância. Assim, empregou-se grandezas elétricas como corrente elétrica, potência elétrica, diferença de potencial para conversão em grandezas fotométricas como luminância e iluminância. A tabela 4 mostra os valores obtidos para as correntes medidas com o método alternativo, as respectivas tensões aplicadas e as correntes medidas com o luxímetro. Os valores de iluminância foram convertidos em luminância para cada imagem padrão teste TG18LN12. Esse teste com base em método alternativo foi realizado nos monitores de Marca Samsung com brilho de $100 \%$.

Tabela 4: Grandezas elétricas para o monitor Samsung B2230, 2,07 MP.

\begin{tabular}{cccccc}
\hline & \multicolumn{6}{c}{ Teste de resposta de luminância e contraste } & & \\
\cline { 2 - 6 } Imagem Teste & $\begin{array}{c}\text { Corrente no } \\
\text { fotodetector } \\
(\mathbf{n A})\end{array}$ & $\begin{array}{c}\text { Tensão } \\
(\mathbf{V})\end{array}$ & $\begin{array}{c}\text { Corrente no } \\
\text { luxímetro } \\
(\mathbf{m A})\end{array}$ & $\begin{array}{c}\text { Iluminância } \\
(\text { lux })\end{array}$ & $\begin{array}{c}\text { Luminância } \\
\left(\mathbf{c d} / \mathbf{m}^{2}\right)\end{array}$ \\
\hline TG18-LN12-01 & 6,0101 & 1,75 & 0,60 & 0,20 & 1,30 \\
TG18-LN12-02 & 6,229 & 1,75 & 0,65 & 0,22 & 1,43 \\
TG18-LN12-03 & 7,350 & 1,75 & 0,70 & 0,24 & 1,56 \\
TG18-LN12-04 & 10,737 & 1,75 & 0,80 & 0,32 & 2,08 \\
TG18-LN12-05 & 17,621 & 1,77 & 1,10 & 0,50 & 3,25 \\
TG18-LN12-06 & 28,264 & 1,79 & 1,40 & 0,70 & 4,55 \\
TG18-LN12-07 & 43,599 & 1,80 & 1,90 & 1,10 & 7,15 \\
TG18-LN12-08 & 62,415 & 1,81 & 2,40 & 1,68 & 10,92 \\
TG18-LN12-09 & 86,805 & 1,83 & 2,80 & 2,16 & 14,04 \\
TG18-LN12-10 & 116,799 & 1,85 & 3,40 & 2,92 & 18,98 \\
TG18-LN12-11 & 149,557 & 1,86 & 4,00 & 3,64 & 23,66 \\
TG18-LN12-12 & 189,234 & 1,83 & 4,60 & 4,48 & 29,12 \\
TG18-LN12-13 & 230,98 & 1,87 & 5,20 & 5,04 & 32,76 \\
TG18-LN12-14 & 273,364 & 1,88 & 5,80 & 6,08 & 39,53 \\
TG18-LN12-15 & 319,327 & 1,89 & 6,60 & 7,28 & 47,33 \\
TG18-LN12-16 & 366,284 & 1,92 & 7,20 & 8,16 & 53,05 \\
TG18-LN12-17 & 409,058 & 1,93 & 7,80 & 8,88 & 57,73 \\
TG18-LN12-18 & 410,724 & 1,93 & 8,00 & 9,04 & 58,73 \\
\hline
\end{tabular}

Nos dados apresentados na curva característica da figura 4 estão implícitas as grandezas elétricas voltagem (V), corrente elétrica (I) e o ângulo sólido equivalente a 0,1538 sr. A quantificação de luz está correlacionada com a de corrente elétrica. Esse procedimento alternativo avalia e classifica o dispositivo de exibição como sendo primário ou secundário. Ao analisar a curva DICOM GSDF da figura 6 e os valores de luminância, comprovou-se um desvio relativo discordante dos limites percentuais de mais ou menos dez por cento do JND, em desacordo com os protocolos de controle de qualidade. Os valores baixos de corrente elétrica medidos nas imagens padrão resultaram em um valor de luminância também baixo. Considerando-se a luminância máxima do monitor, o monitor SAMSUNG S20B300 (figura 6) foi classificado como secundário, como era de se esperar. Nessa avaliação o tempo de uso do dispositivo foi levado em consideração, pois monitores comuns não devem ser usados para prescrição de laudo médico, sendo apenas para visualização de imagens médicas. 


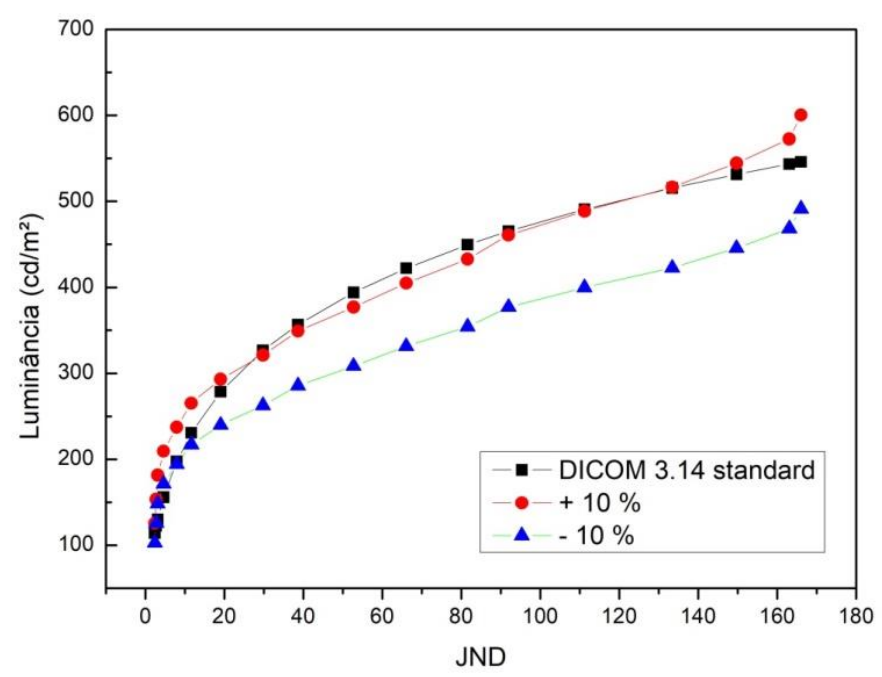

Figura 6: Curva característica DICOM GSDF da luminância versus JND com brilho de 100 \% para o monitor SAMSUNG S20B300.

O controle de qualidade nos dispositivos de exibição para o diagnóstico médico foi ainda verificado pelo teste de uniformidade de luminância. De maneira geral, para os monitores de marca Barco os valores de luminância nas quatro bordas e no centro das imagens teste TG18- UNL80 e TG18-UNL10 apresentaram os percentuais previstos para luminância máxima. Nesse teste, observou-se uma variação da intensidade luminosa na tela do monitor para um mesmo tom de cinza. Quanto aos valores de luminância nas regiões das imagens padrões teste, tanto para os monitores de marca Barco e Samsung, os resultados apresentaram uma variação de luminância no centro da imagem em relação às bordas, que não ultrapassou 30\%, significando que os resultados estão em conformidade o estabelecido nos protocolos de controle de qualidade. De acordo com esse teste, os monitores da marca Barco são considerados primários e os da Samsung são considerados secundários.

\section{CONCLUSÃO}

Neste artigo foram analisados, de maneira quantitativa e qualitativa, os dispositivos de exibição, utilizando-se grandezas fotométricas e, alternativamente, grandezas elétricas. A avaliação das imagens testes do protocolo AAPM [5], visualizadas nos monitores, possibilitaram classificar esses dispositivos de exibição de imagens como adequados ao diagnóstico médico (primários) ou apenas para visualização de imagens médicas (secundários). Alternativamente, empregou-se para avaliação da qualidade os monitores um luxímetro digital calibrado e seu valor de ângulo sólido, já calculado para conversão de unidade de iluminância em luminância. Constatou-se que tal método alternativo pode ser útil para esse tipo de avaliação na indisponibilidade de um fotômetro.

Os testes de resolução, ruído e reflexão interna permitiram classificar os monitores com base na acuidade do sistema visual humano. Em relação ao teste de resposta de luminância e contraste, de maneira geral, com a curva característica se verificou que os monitores estavam de acordo com os protocolos de controle de qualidade, considerando-se os valores da razão de luminância e razão de contraste. Os resultados experimentais obtidos com grandezas elétricas, convertidas em grandezas fotométricas comprovaram que monitores comuns não devem ser usados para o diagnóstico médico, apenas para visualização de imagens médicas. 


\section{AGRADECIMENTOS}

O presente trabalho foi realizado com apoio da Coordenação de Aperfeiçoamento de Pessoal de Nível Superior - Brasil (CAPES) e do Laboratório de Instrumentação Nuclear do Centro Regional de Ciências Nucleares do Nordeste da Comissão Nacional de Energia Nuclear.

\section{REFERÊNCIAS BIBLIOGRÁFICAS}

1. Nema, PS.3.14-2006 Digital Imaging and Communications in Medicine (DICOM Part14: Grayscale Standard Display Function). National Electrical Manufacturers Association, 2006.

2. Barten P. Physical model for the contrast sensitivity of the human eye. Proc. SPIE. PIE Human Vision, Visual Processing and Digital Display III. 1666; 1992:57-72.

3. Barten P. Spatio-temporal model for the contrast sensitivity of the human eye and its temporal aspects. Proc SPIE, Human Vision, Visual Processing, and Digital Display IV. 1993;1913:2-14, doi: $10.1117 / 12.152690$

4. Pires RP. Software gerenciador de base de dados e imagens radiográficas e imagens radiológicas para avaliação de monitores [tese]. São Paulo: Universidade Federal de São Paulo: Escola Paulista de Medicina; 2007. $138 \mathrm{p}$.

5. American Association of Physicists in Medicine. (Assessment of Display Performance for Medical Imaging Systems). Report 03; 2005.

6. Protocolo Espanhol de Controle de Qualidade no Radiodiagnóstico: Protocolo Español de Control de Calidad em Radiodiagnóstico. Revisión 2011, Senda Editorial: S.A; 2012.

7. Protocol for the Quality Control of the Physical and Technical Aspects of Digital Breast Tomosynthesis Systems, R. van Engen; 2016. 55 p.

8. Favero MS, Goulart AS. Controle de Qualidade dos monitores de interpretação de imagens radiológicas digitais: Uma revisão. Rev Bras Fis Med. 2016;10(1):13-16, doi:10.29384/rbfm

9. Pinto M, Pedro M, Santos A, Saraiva A. Display quality control based on luminance measurements in post-processing units at imaging diagnosis centers. Radiol Bras. 2012;45(1):29-34, doi:10.1590/S010039842012000100008

10. Mesquita BR. Controle de qualidade em dispositivos de exibição de imagens utilizados em radiodiagnóstico empregando o padrão GSDF e JND [dissertação]. São Cristovão: Universidade Federal de Sergipe; 2017, 139 p.

11. Ekpo EU, Mcentee MF. An evaluation of performance characteristics of primary display devices. J Digit Imaging. 2016; 175-182, doi:10.1007/s10278-0159831-3

12. Ribeiro LD, Furquim TAC. Estudo do desempenho de monitores LCD em radiologia com imagem digital. Rev Bras Fis Med. 2010;4(2):27-30. 